\title{
A Graphene-Based THz Wave Duplexer and Filter: Switching via Gate Biasing
}

\section{AmirAli Mohammad Khani ( $\nabla$ a.m.khani1986@gmail.com )}

Islamic Azad University Saveh Branch https://orcid.org/0000-0001-5171-8707

\section{Research Article}

Keywords: Graphene, Thin layers, THz, Optical Duplexer

Posted Date: December 29th, 2021

DOI: https://doi.org/10.21203/rs.3.rs-1091948/v1

License: (c) (i) This work is licensed under a Creative Commons Attribution 4.0 International License. Read Full License

Version of Record: A version of this preprint was published at Optik on February 1st, 2022. See the published version at https://doi.org/10.1016/j.ijleo.2021.168432. 


\title{
A Graphene-Based THz Wave Duplexer and Filter: Switching via Gate Biasing
}

\author{
Amir Ali Mohammad Khani \\ Department of Electrical Engineering, College of Technical and Engineering, Saveh Branch, Islamic Azad \\ University, Saveh, Iran.
}

\begin{abstract}
:
This work introduces a Graphene-based multi-layer reconfigurable device as a wave duplexer in the $\mathrm{THz}$ frequency range. Adjusting transmitting and reflecting parts of incident waves alongside controlling absorption provides the interesting capability to select target waves in different frequencies. The proposed device includes periodic graphene patterns on both sides of silicon dioxide as substrate. Additionally, the patterns are biased differently compared to conventional patterns which makes it possible to achieve two distinct behaviors versus frequency. Exploiting equivalent circuit models (ECM) for graphene and dielectric, the whole device is modeled by passive RLC circuits. According to simulation results, the proposed device can transmit and reflect incident $\mathrm{THz}$ waves at desired frequencies in $0.1 \mathrm{THz}$ to $30 \mathrm{THz}$ which makes it an ideal candidate for manipulating $\mathrm{THz}$ waves in terms of transmission and reflection.
\end{abstract}

Keywords: Graphene, Thin layers, THz, Optical Duplexer

\section{Introduction:}

In 2002, a wide range of $0.1 \mathrm{THz}-30 \mathrm{THz}$ (including wavelengths between $10 \mu \mathrm{m}-3 \mathrm{~mm}$ ) was introduced as the "THz gap". This frequency spectrum was introduced to create devices that could act as a source or tracker. In 2005, the THz gap was defined as the spectrum of the region $0.1 \mathrm{THz}-10 \mathrm{THz}$ (corresponding to the wavelength $10 \mu \mathrm{m}-3 \mathrm{~mm}$ ). The definition of terahertz has changed over the past 18 years. However, the definition has been in the range of $0.1 \mathrm{THz}-10 \mathrm{THz}$ in most cases [1]. This band has been considered by many researchers, which can be attributed to the unique properties of this frequency range. In astronomy, for example, the terahertz emission and absorption spectra have been used for space exploration. In medicine, terahertz radiation has been used to image the human body (with lower risks compared to X-rays) [2-3]. In addition, terahertz radiation 
provides carrier frequencies in telecommunication protocols much higher than those used in current communication systems (wireless and commercial). It has also been used to increase data transmission capacity for short-range (short-distance) communications. The various applications of the terahertz band have made this area one of the most interesting research topics [4-8]. Implementing such applications requires materials to realize terahertz devices. Researchers interested in the terahertz band have studied the properties of materials with different dimensions. In terms of common three-dimensional materials, the electrical conductivity and conductivity of silica and gold have been investigated in the terahertz band. In addition, a range of two-dimensional materials such as graphene, phosphorene, and silicon have also been investigated [9].

Among these two-dimensional materials, graphene has been the subject of research in various fields due to its unique mechanical, thermal, and electromagnetic properties. One of the attractive properties of graphene as a semiconductor-metal material is its unique conductivity, which can be controlled by external voltage bias or static magnetic bias. Now, along with the terahertz frequency band and graphene material, we need a way to model and realize such devices. To help design accurate structure, it is essential to provide numerical modeling methods that take into account the properties of graphene [9], [30-34].

One of the simplification approaches to solving electromagnetic problems is to describe the elements as circuit elements. In the meantime, relatively accurate equations are presented to describe the graphene layer.

In 2014 and 2015, references [10] and [11] presented efficient circuit models for graphene nano-strips and graphene nano-disks, respectively, which consider the effects of physical parameters such as the geometry and electron relaxation time along with the effects of bias voltage has taken into account.

According to many previous works, the accuracy of the circuit model performance has been compared with numerical methods, most of which report a very good agreement with the slight error of the circuit model method versus numerical methods [10], [11]. Based on this, it can be concluded that the circuit model approach can be a substitute for time-consuming and complex numerical methods. In this regard, three graphene patterns are focused. 
Nano-strips, nano-disks, and graphene continuous plates are modeled in series with resistor-inductor-capacitor circuit elements. This modeling, along with knowing the dielectric circuit model, leads to the calculation of the structure impedance. Matching this impedance with the impedance of the outer space of the structure means the transfer of maximum power from the external environment to the structure (maximum power in an absorber means complete absorption in the structure). In other words, the impedance adaptation is equivalent to the full absorption at the frequency at which this adaptation occurred. Thus, this approach paves the way for the design of graphene-based devices by simplifying an electromagnetic phenomenon to the level of an impedance matching problem [18-23].

In this way, here two graphene patterns on both sides of dielectric form a reconfigurable device that can select the desired reflection and/or transmission waves.

Section 2 describes the proposed device in detail with corresponding circuit representation. Also, section 3 provides simulations results while section 4 concludes the work as the conclusion.

\section{Proposed Device:}

The proposed device is illustrated in Fig. 1, includes two periodic arrays of Graphene on both sides of a dielectric. Each Graphene pattern consists of a triple-bias scheme. According to [14], bias equalities can force patterns to experience different periods, and consequently, a degree of freedom is provided to adjust device reactions in desired frequencies. In addition, based on [12-15], [35-37], circuit representation of graphene patterns is reported with excellent accuracy compared to full numerical simulations. According to Fig. 1, the incident wave divides into three parts: Transmitted, Absorbed, and Reflected parts. By minimizing the absorption part, interesting functions can be realized via transmission and reflection parts. In this way, the proposed structure can act similar to a duplexer which can transmit or reflect target waves. 
Fig. 2 shows Graphene patterns and biasing schematics. According to Fig. 2, The pattern parts are connected via metal wire. Also, graphene bridges or narrow ribbons can be used instead of metal wire. Here yellow ribbons are illustrated for visual effect simplicity, while the usual scenario is using graphene nanoribbons to connect patterns part. The point is that width of exploited connecting elements (metal or graphene) must be smaller operational wavelengths.

Fig. 3 presents the circuit description for the device components. So, the entire device can be described as a unique impedance. The circuit elements are arranged in such a way that the graphene layers are parallel and the series dielectric layer is placed. This type of arrangement is due to the description of the transmission line theory for point loads. In other words, graphene is assumed to be a very thin layer at the load point.

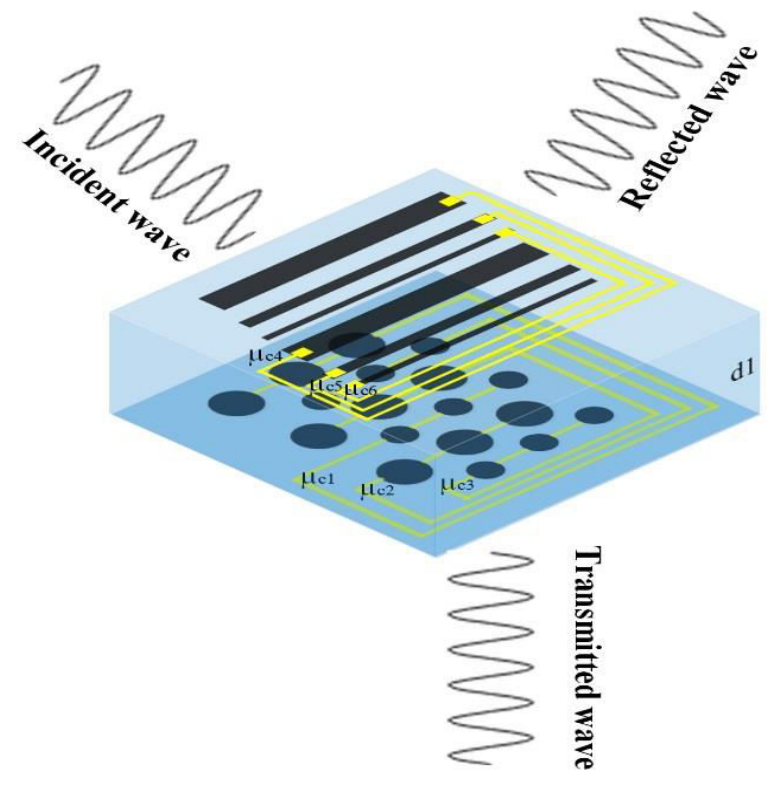

Fig. 1. Proposed device. $h=13 \mu m, r=6.4 \mu m, \mathrm{P}=16 \mu m, \mathrm{~W}=3.4 \mu m, \mathrm{D}=8.5 \mu \mathrm{m}$. $h, r, \mathrm{P}, \mathrm{W}$, and $\mathrm{D}$ are dielectric thickness, disk radius, disk period, ribbons width, and ribbons period respectively. It should be noted that the illustration is symbolic while all disks are the same in shape and all ribbons have equal width. Also, based on [15], periods of disks and ribbons can vary be corresponding to bias equalities. 


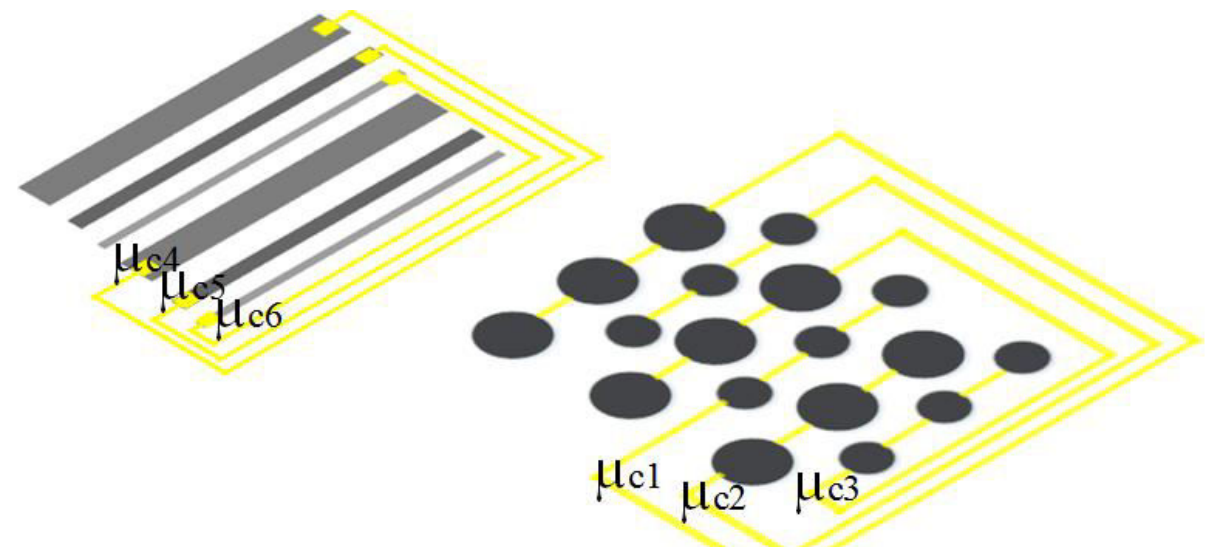

Fig. 2. Graphne patterns and biasing schematics.

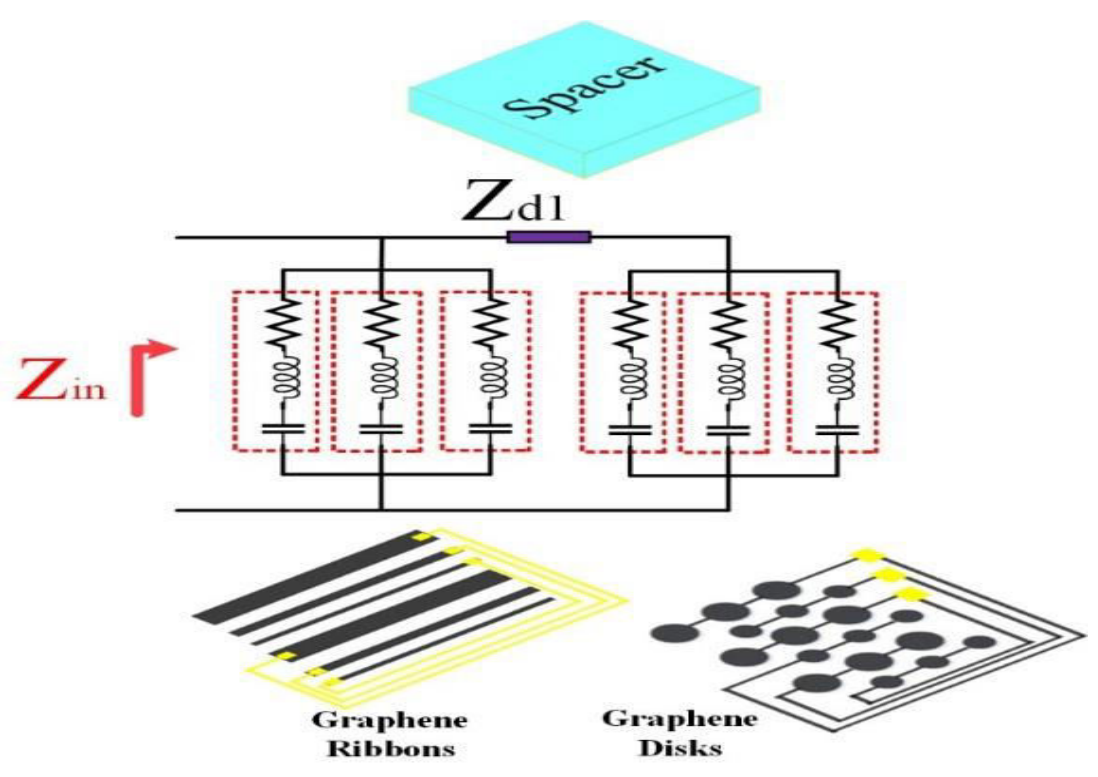

Fig. 3. Equivalent circuit model for the proposed device. The dielectric $\mathrm{SiO}_{2}$ is modeled as pure imaginary impedance while each Graphene pattern is modeled via three parallel RLC branches, each RLC branch is corresponding to one of the existing biases. Six possible biases in Fig.1 can adjust six existing RLC branches.

According to [10] and [14], each pattern can be modeled via a resistor, inductor, and capacitor. These elements are related to some physical constants and geometrical sizes as bellow: 
Graphene Ribbons :

$R_{n}=\frac{D}{S_{n}^{2}} \frac{\pi \hbar^{2}}{e^{2} E_{F} \tau}$

$L_{n}=\frac{D}{S_{n}^{2}} \frac{\pi \hbar^{2}}{e^{2} E_{F}}=R_{n} \tau$

$C_{n}=\frac{S_{n}^{2}}{D} \frac{2 \varepsilon_{\text {eff }}}{q_{n}}$

Graphene Disks :

$R_{n}=\frac{P^{2} K_{n}}{\pi S_{n}^{2}} \frac{\hbar^{2}}{e^{2} E_{F} \tau}$

$L_{n}=\frac{P^{2} K_{n}}{\pi S_{n}^{2}} \frac{\hbar^{2}}{e^{2} E_{F}}=R_{n} \tau$

$C_{n}=\frac{\pi^{2} S_{n}^{2}}{P^{2} K_{n}} \frac{\varepsilon_{\text {eff }}}{q_{1 n}}$

where the definition of parameters of Eq. (1) and Eq. (2) are reported in Table 1:

Table 1. Definition of parameters.

\begin{tabular}{|c|c|c|c|}
\hline parameter & definition & parameter & definition \\
\hline$R_{n}$ & $\begin{array}{c}\text { Equivalent resistance } \\
\text { of series branch }\end{array}$ & $\varepsilon_{\text {eff }}=\frac{\varepsilon_{1}+\varepsilon_{2}}{2}=\frac{n_{1}^{2} \varepsilon_{0}+n_{2}^{2} \varepsilon_{0}}{2}$ & Effective permittivity \\
\hline$L_{n}$ & $\begin{array}{c}\text { Equivalent inductor of } \\
\text { series branch }\end{array}$ & $\varepsilon_{1}$ & $\begin{array}{l}\text { Permittivity of first } \\
\text { substance }\end{array}$ \\
\hline$C_{n}$ & $\begin{array}{c}\text { Equivalent capacitor } \\
\text { of series branch }\end{array}$ & $\varepsilon_{2}$ & $\begin{array}{c}\text { Permittivity of second } \\
\text { substance }\end{array}$ \\
\hline$P$ & $\begin{array}{l}\text { Period of graphene } \\
\text { disks }\end{array}$ & $\varepsilon_{0}=8.85 \times 10^{-12} \frac{F}{m}$ & $\begin{array}{c}\text { Free space } \\
\text { permittivity }\end{array}$ \\
\hline$D$ & $\begin{array}{l}\text { Period of graphene } \\
\text { ribbons }\end{array}$ & $E_{F}=\frac{\tau e v_{F}^{2}}{\mu}=e \mu_{c}$ & Fermi energy level \\
\hline$K_{n} \cong\left(n-\frac{1}{4}\right) \frac{\pi}{\omega}$ & Eigen value & $\tau=\frac{\mu E_{F}}{e v_{F}^{2}}$ & $\begin{array}{l}\text { Electron relaxation } \\
\text { time } \\
\end{array}$ \\
\hline$S_{n}$ & Eigen function & $\mu$ & Electron mobility \\
\hline$q_{n}$ & Eigen value & $\mu_{c}$ & Chemical potential \\
\hline
\end{tabular}




\begin{tabular}{|c|c|c|c|}
\hline$q_{1 n}$ & Eigen value & $e=1.602 \times 10^{-19} \mathrm{C}$ & Electron charge \\
\hline$v_{F}=10^{6} \frac{\mathrm{m}}{\mathrm{s}}$ & $\begin{array}{c}\text { Fermi velocity of } \\
\text { graphene }\end{array}$ & $\hbar=6.58 \times 10^{-16} \mathrm{eV} \mathrm{s}$ & $\begin{array}{c}\text { Reduce Plank } \\
\text { Constant }\end{array}$ \\
\hline \multicolumn{2}{|c|}{$\varepsilon_{\text {dielectric }}=n_{\text {dielectric }}^{2} \times \varepsilon_{0}=(1.45)^{2} \times 8.85 \times 10^{-12}=18.6 \times 10^{-12} \frac{F}{m}$} & $\begin{array}{c}\text { Permittivity of } \\
\text { dielectric }\end{array}$ \\
\hline
\end{tabular}

To obtain $q_{n}$, and $q_{1 n}$, physical parameter such as $r, \mathrm{P}, W$, and $D$, must be designed and then referred to Table 2 .

Table 2. the value of $q_{n}$ and $q_{1 n}$ based on the geometry of the proposed device.

\begin{tabular}{|c|c|c|c|c|c|c|c|c|c|}
\hline$\frac{2 r}{P}$ & 0.1 & 0.2 & 0.3 & 0.4 & 0.5 & 0.6 & 0.7 & 0.8 & 0.9 \\
\hline Graphene Disks : $\mathrm{r} q_{11}$ & 0.539 & 0.536 & 0.533 & 0.530 & 0.527 & 0.499 & 0.472 & 0.444 & 0.417 \\
\hline$\frac{W}{D}$ & 0.1 & 0.2 & 0.3 & 0.4 & 0.5 & 0.6 & 0.7 & 0.8 & 0.9 \\
\hline Graphene Ribbonss: $q_{1} \frac{W}{\pi}$ & 0.734 & 0.725 & 0.710 & 0.689 & 0.658 & 0.620 & 0.571 & 0.507 & 0.420 \\
\hline
\end{tabular}

According to [16] and Fig. 3, impedance load tends to graphene disks $\left(Z_{L}=Z_{\text {graphene-disks }}\right)$. So, $Z_{1}$ impedance sees to graphene disks at the end of the line. Therefore, $Z_{1}$ calculated by Eq. (3). Then Eq. (4) is obtained based on the transmision line concept [16]. Also, the dielectric can be modeled via (5). And the input impedance is calculated as below:

$$
\begin{aligned}
& Z_{1}=Z_{\text {graphene-disk }} \\
& Z_{2}=Z_{d} \frac{Z_{\text {graphene-disk }}+j Z_{d} \cdot \tan \left(\beta_{d} h\right)}{Z_{d}+j Z_{\text {graphene-disk }} \cdot \tan \left(\beta_{d} h\right)} \\
& Z_{d}=\frac{Z_{0}}{n_{d}} \\
& Z_{\text {in }}=Z_{2} \| Z_{\text {graphene-ribbon }}=\frac{Z_{2} \cdot Z_{\text {graphene-ribbon }}}{Z_{2}+Z_{\text {graphene-ribbon }}}
\end{aligned}
$$


where the definition of parameters of Eq. (3) to Eq. (6) are reported in Table 3:

Table 3. Definition of parameters.

\begin{tabular}{|c|l|}
\hline Parameter & \multicolumn{1}{c|}{ Definition } \\
\hline$\beta_{d}$ & The wave propagation constant in the dielectric substrate. \\
\hline$h$ & The dielectric thickness. \\
\hline$Z_{0}$ & The free space impedance and equal to $120 \pi \Omega$. \\
\hline$n_{d}$ & Refractive index of dielectric. \\
\hline
\end{tabular}

Since the main goal in designing the proposed device is to achieve the maximum transmission and/or reflection against bias changing, calculating device impedance is inevitable to verify the matching concept and knowing absorption, transmission, and reflection percentage in the desired frequency range. In another word, if $Z_{\text {in }} \approx Z_{0} \approx 120 \pi$, the device can achieve maximum absorption while far values of input impedance compared to $120 \pi$, minimized absorption and increase total transmission and reflection.

Accurate calculation of Eq. (6) is very easy numerically and very complex symbolically (parametrically). For instance, the exact input impedance of the structure can be seen in the MATLAB output, which indicates the complexity and nonlinearity of this impedance. Due to the high volume of calculations in achieving Eq. (6), this impedance is not reported in the text of this article.

Based on Eq. (6), the input impedance of the proposed structure is calculable versus frequency. All parameters in the proposed structure have their effects on the impedance. So finding the impedance sensitivity to parameters is interesting to obtain distinct responses. The point is that physical parameters cannot alter after fabrication while chemical potentials can change in a limited range. So logical approach is to search for different responses against different chemical potentials which meet the desired scenario. In this way, a simple algorithm is developed to relatively optimize input impedance versus bias values. To set criteria two functions are defined by Eq. (7): 
Multi-Band :

$C . F=\frac{A_{1}+A_{2}+\ldots+A_{n-1}+A_{n}}{n}$

$A_{n} \rightarrow$ Absorption in $f_{n}$

$n \rightarrow$ Number of Frequencies

Width-Band :

$C . F .=\frac{1}{f_{H}-f_{L}} \int_{f_{L}}^{f_{H}} 1-\mid$ gamma $\left.\right|^{2} d f$

In this way, with an iterative algorithm, two sets of chemical potentials are obtained as Mode A and Mode B which are tabulated in the next section.

\section{Simulation Results:}

According to Eq. (8), changing gate bias leads to changing chemical potential which forces the device to react differently [17]. In this way, two operational modes are defined with corresponding chemical potentials, tabulated in Table 4.

$V_{g}=\frac{\mu_{c}^{2} e h}{\hbar^{2} v_{f}^{2} \varepsilon_{0} \varepsilon_{\text {dielectric }} \pi}$

Where $V_{g}, \varepsilon_{r}, \varepsilon_{0}, h$, and $v_{f}$ are respectively the gate voltage, the permittivity of the dielectric substrate, the vacuum permittivity, the thickness of the dielectric substrate, and the Fermi velocity. The values of the parameters of Eq. (8) are given in Table 1.

The described device is simulated via two sets of bias values. The corresponding chemical potentials for each bias set are tabulated in Table 4.

Table 4. Two sets of bias values for the proposed device.

\begin{tabular}{|l|c|c|}
\cline { 2 - 3 } \multicolumn{1}{c|}{} & First operational mode $(\mathrm{eV})$ & Second operational mode $(\mathrm{eV})$ \\
\hline$\mu_{c 1}$ & 0.45 & 0.85 \\
\hline$\mu_{c 2}$ & 0.60 & 0.66 \\
\hline$\mu_{c 3}$ & 0.85 & 0.15 \\
\hline$\mu_{c 4}$ & 0.15 & 0.45 \\
\hline$\mu_{c 5}$ & 0.45 & 0.35 \\
\hline$\mu_{c 6}$ & 0.60 & 0.90 \\
\hline
\end{tabular}


The first operational mode named Mode-A shows a transmission coefficient of about $85 \%$ from $1 \mathrm{THz}$ to $3.5 \mathrm{THz}$ which can be considered as a relatively wide band filter while the second mode of operation (Mode-B) expresses two transmission peaks at $1.2 \mathrm{THz}$ and 6.5 THz. Considering transmission, reflection, and absorption values versus frequency simultaneously verifies that the proposed device can efficiently be used as a $\mathrm{THz}$ wave duplexer for switching between transmitting or reflecting $\mathrm{THz}$ waves via changing external bias voltages. Fig. 4 is reported to show transmission, reflection, and absorption simultaneously versus frequency. Additionally, Fig. 5 is reported to investigate device behavior versus geometrical variations include graphene disks, Graphene ribbons, and dielectric. It should be noted that the exploited dialectic is $\mathrm{SiO}_{2}$ with a refractive index equal to 1.45 . Also, the value of electron relaxation time is considered $0.1 \mathrm{ps}$, and the thickness of Graphene has assumed about $1 \mathrm{~nm}$ approximately about three carbon atoms regarding considerations of non-idealities in the fabrication method. Otherwise, ideal Graphene is a two-dimensional material with a thickness of about $0.34 \mathrm{~nm}$.

Similarly, the second mode of operation is reported in Fig. 6 and Fig. 7.

According to simulation results, both operational modes are in an acceptable condition against geometrical variations while the device can be switched easily between two modes just by setting bias values. 


\section{Mode-A:}

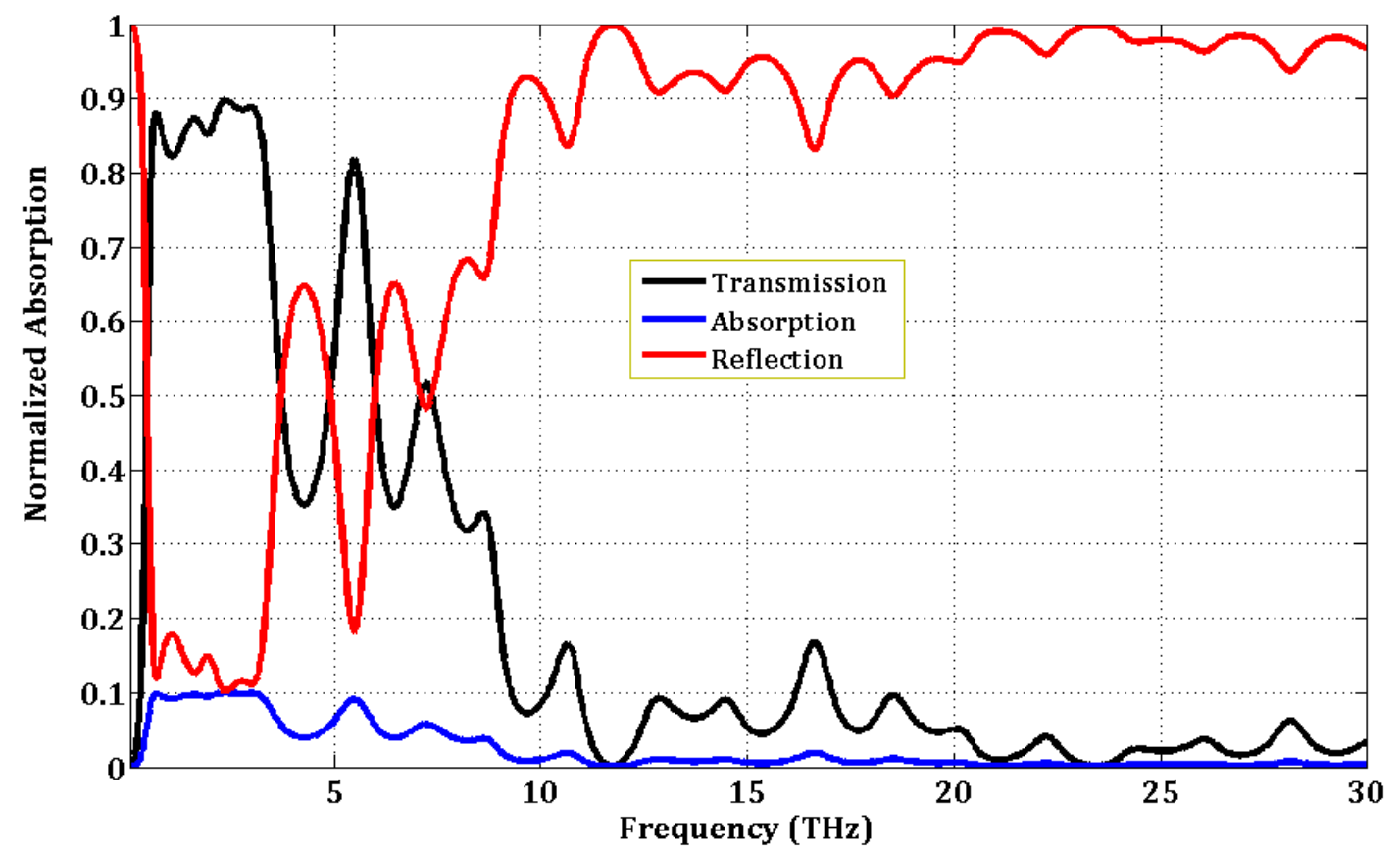

Fig. 4. Transmission, Reflection, and Absorption coefficients versus frequency for the first mode of operation.

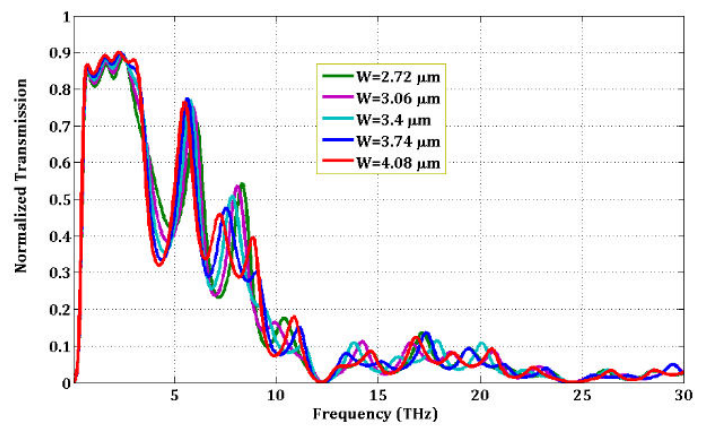

(A)

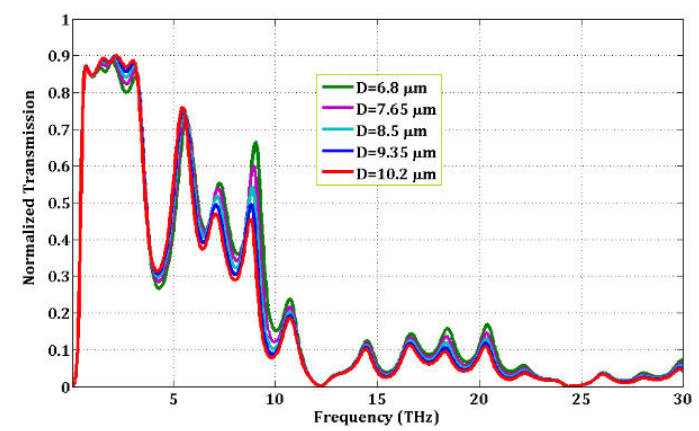

(C)

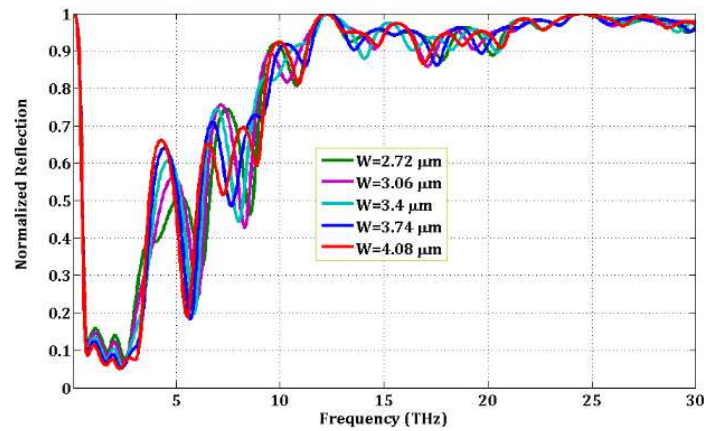

(B)

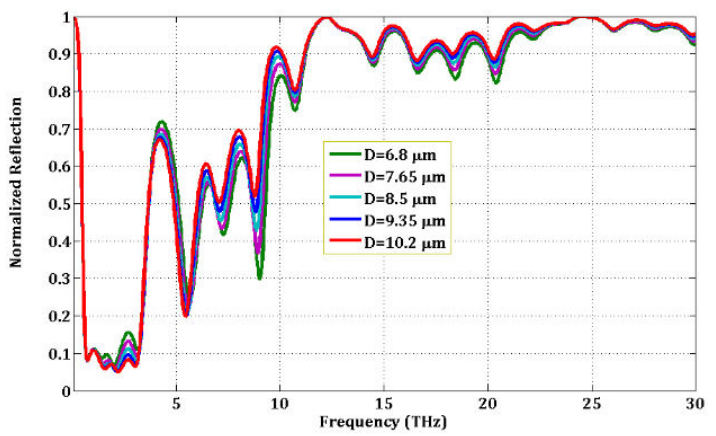

(D) 


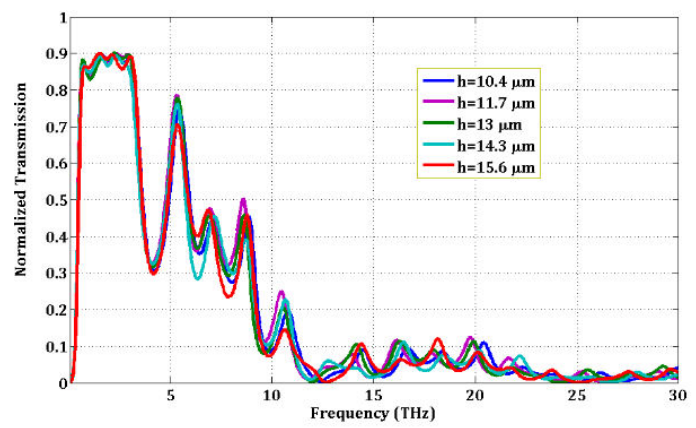

(E)

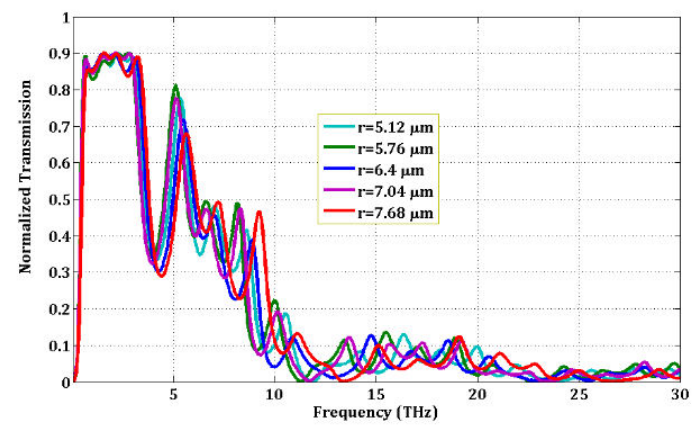

(G)

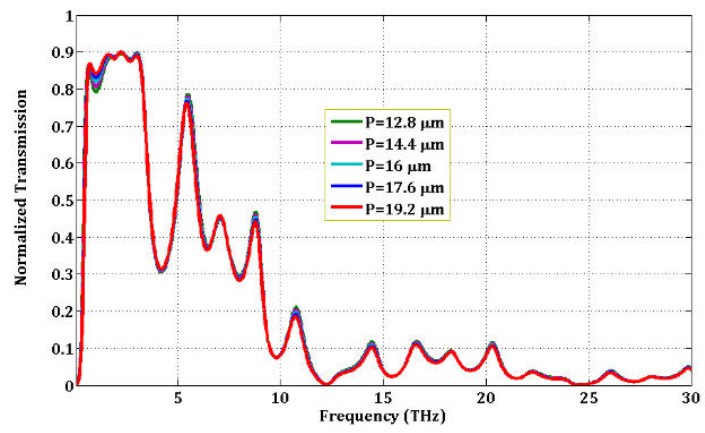

(I)

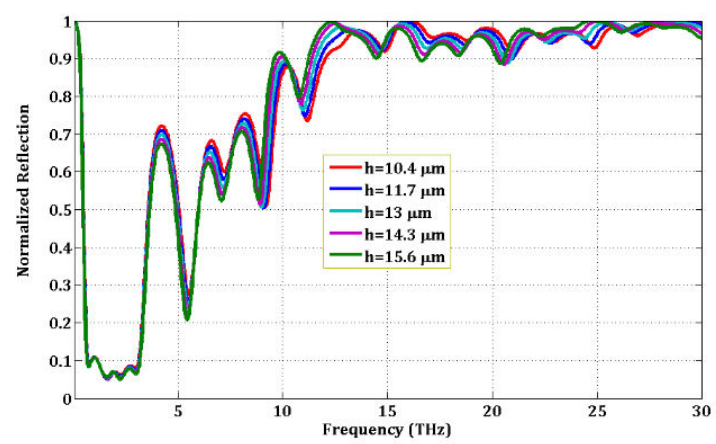

(F)

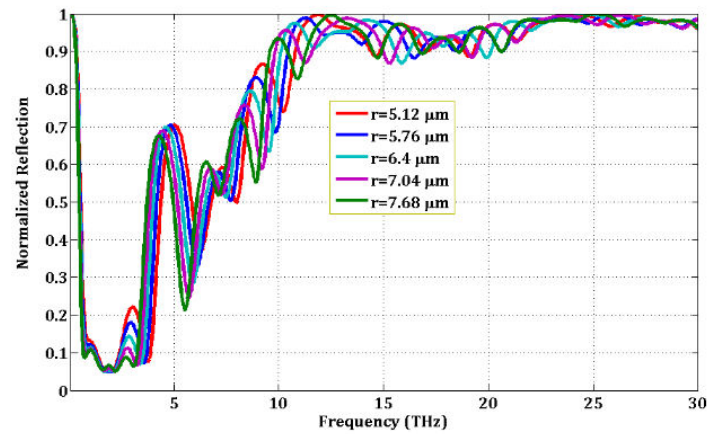

$(\mathrm{H})$

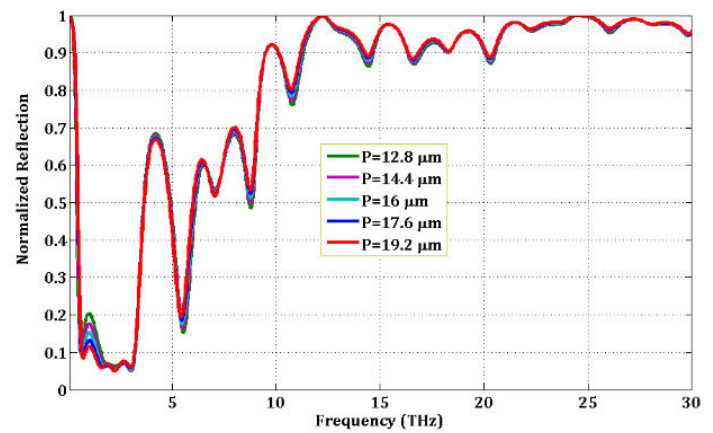

$(\mathrm{K})$

Fig. 5. Sensitivity against geometrical variation. A: Transmission for different ribbons width, B: Reflection for different ribbons width, C: Transmission for different ribbons period, D: Reflection for different ribbons period, E: Transmission for different height of dielectric, F: Reflection for different height of dielectric, G: Transmission for different disks radius, H: Reflection for different disks radios, I: Transmission for different disks period, K: Reflection for different disks period. 


\section{Mode-B}

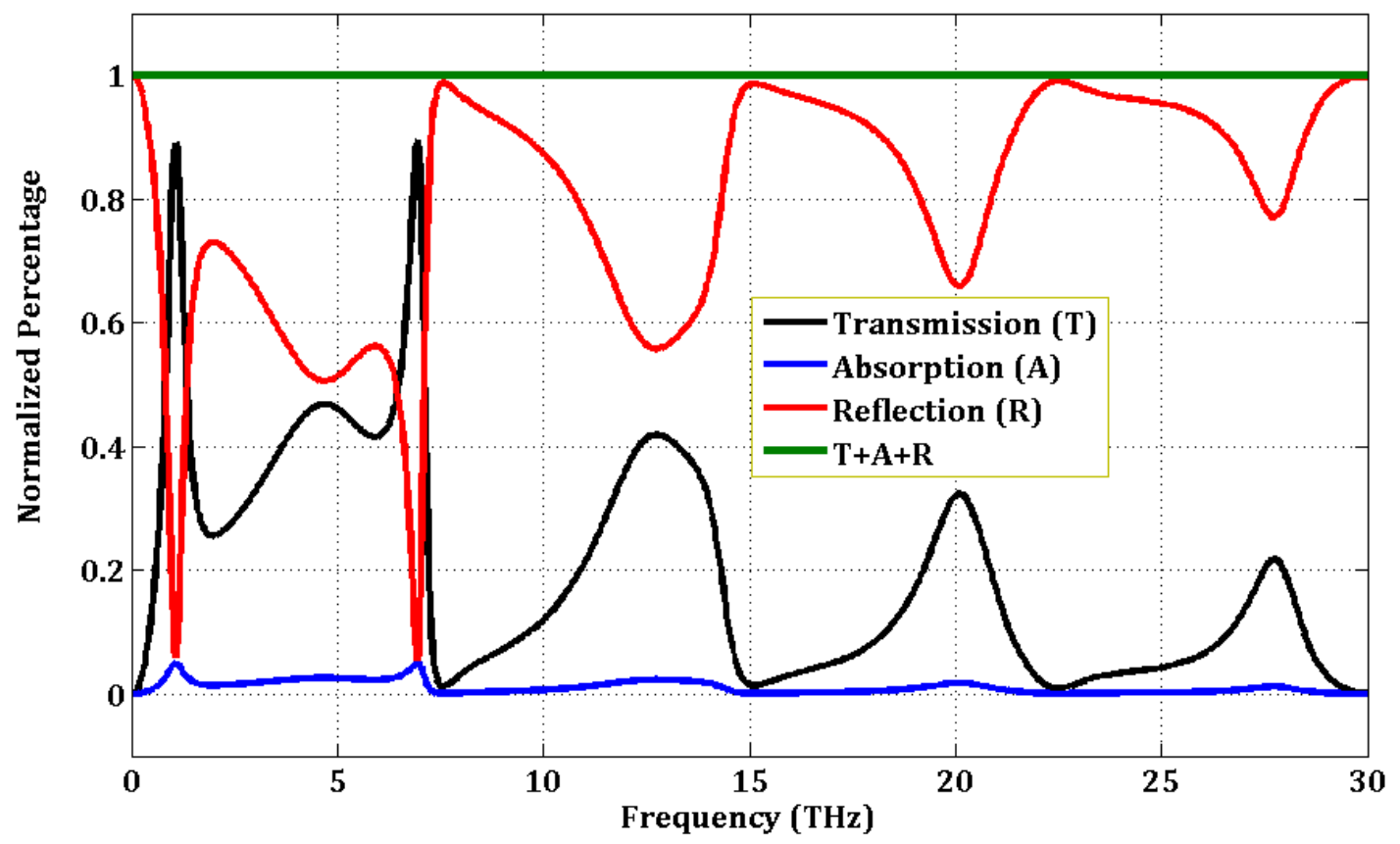

Fig. 6. Transmission, Reflection, and Absorption coefficients versus frequency for the second mode of operation.

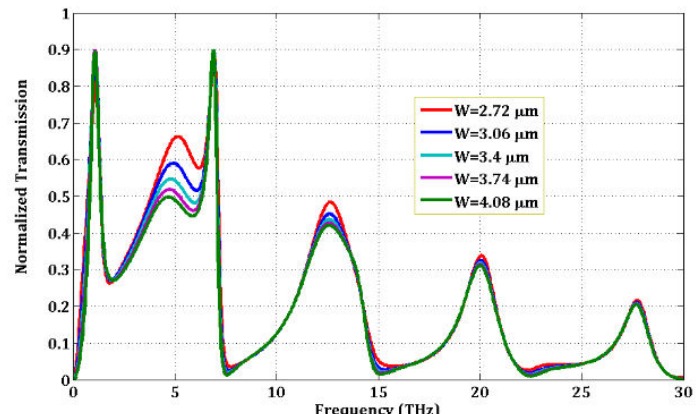

(A)

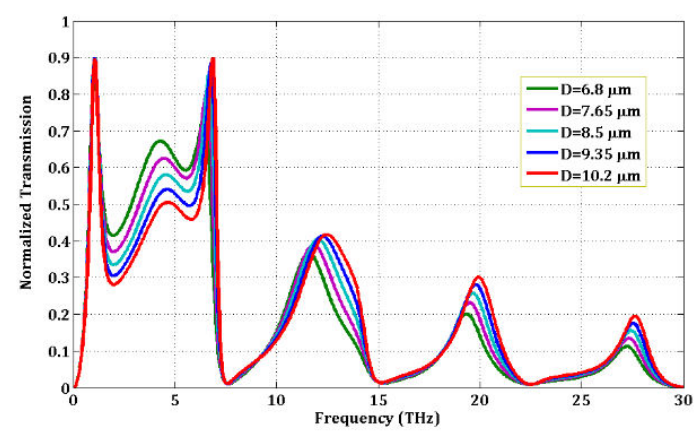

(C)

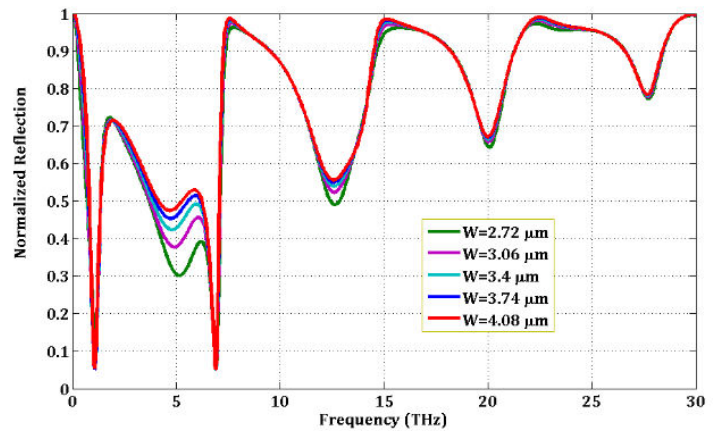

(B)

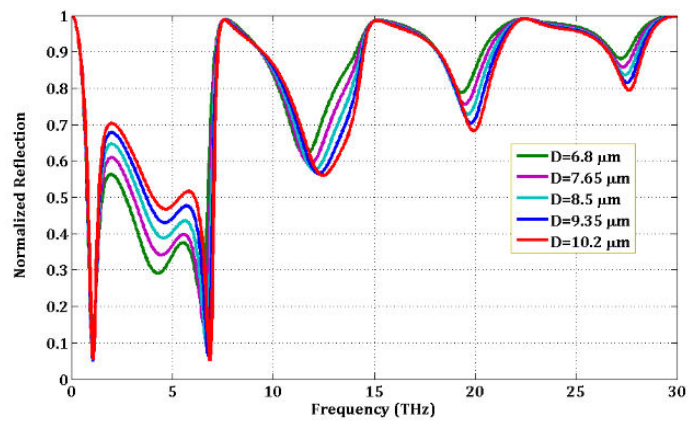

(D) 


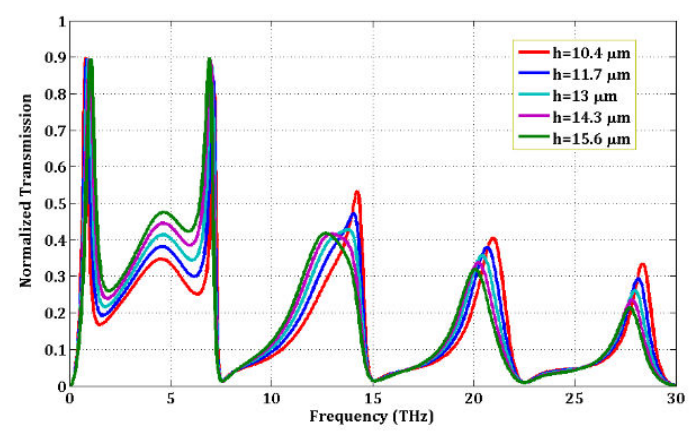

(E)

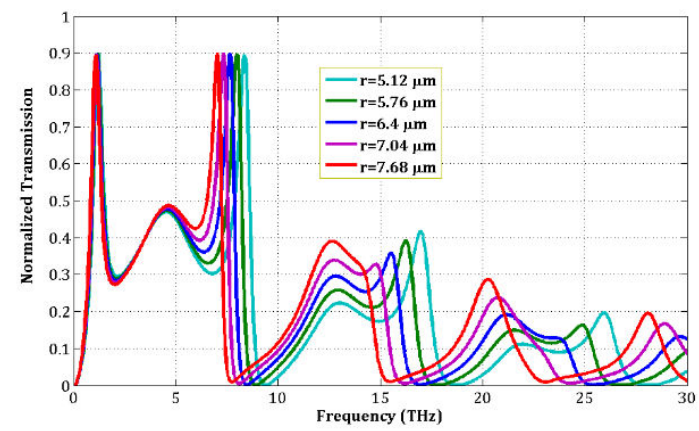

(G)

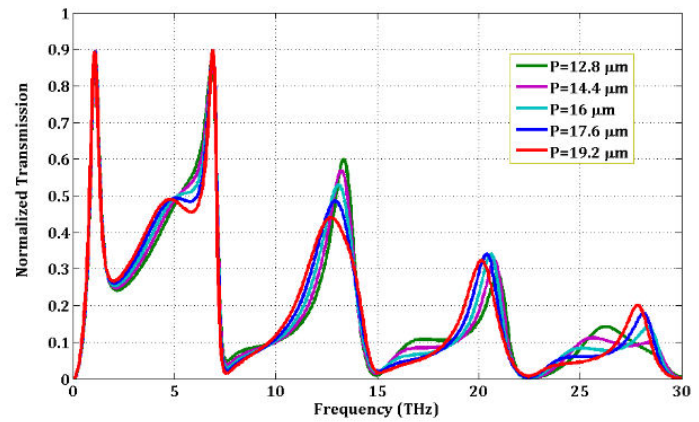

(I)

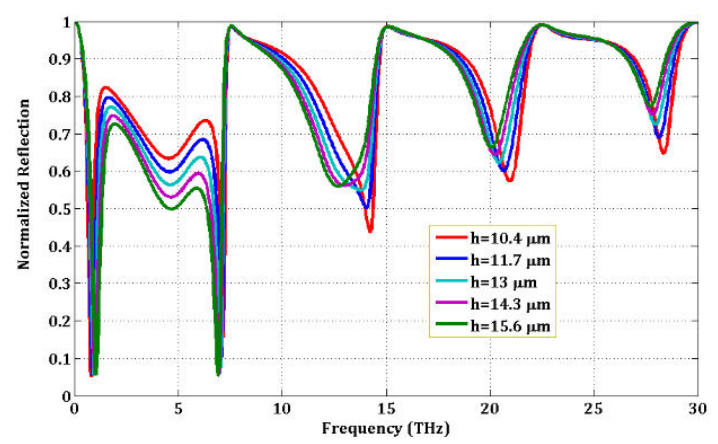

(F)

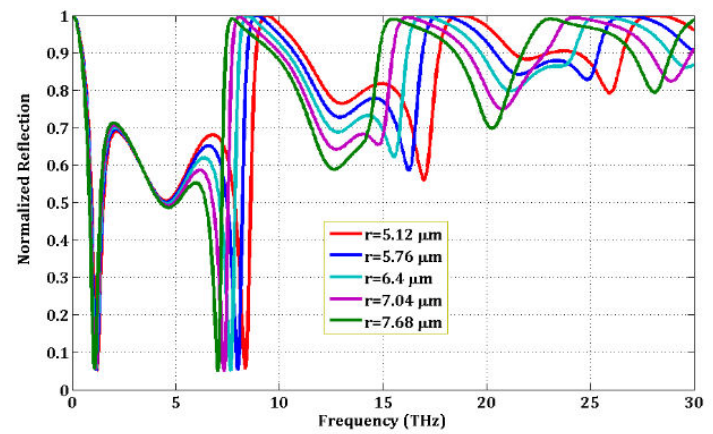

$(\mathrm{H})$

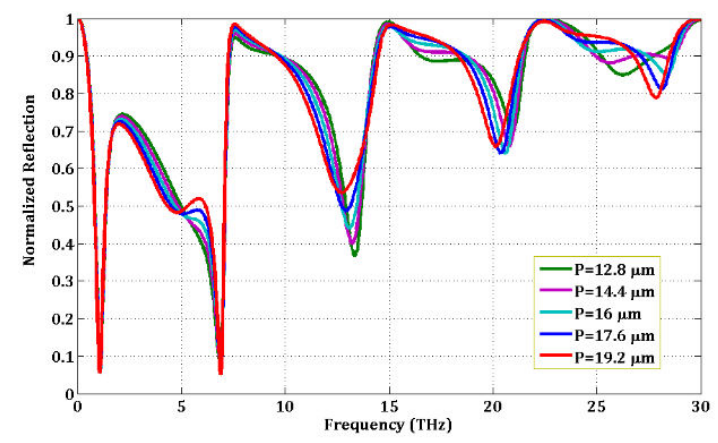

$(\mathrm{K})$

Fig. 7. Sensitivity against geometrical variation (Mode-B). A: Transmission for different ribbons width, B: Reflection for different ribbons width, C: Transmission for different ribbons period, D: Reflection for different ribbons period, E: Transmission for different height of dielectric, F: Reflection for different height of dielectric, G: Transmission for different disks radius, $\mathrm{H}$ : Reflection for different disks radios, I: Transmission for different disks period, K: Reflection for different disks period.

At last but not least, further verification via full-wave simulations is performed by reporting both transmission and reflection versus different incident angles. According to 
these Fig. (8) to Fig. (11) the ECM results are perfectly matched by full-wave simulation results obtained via Finite Element Method (FEM).

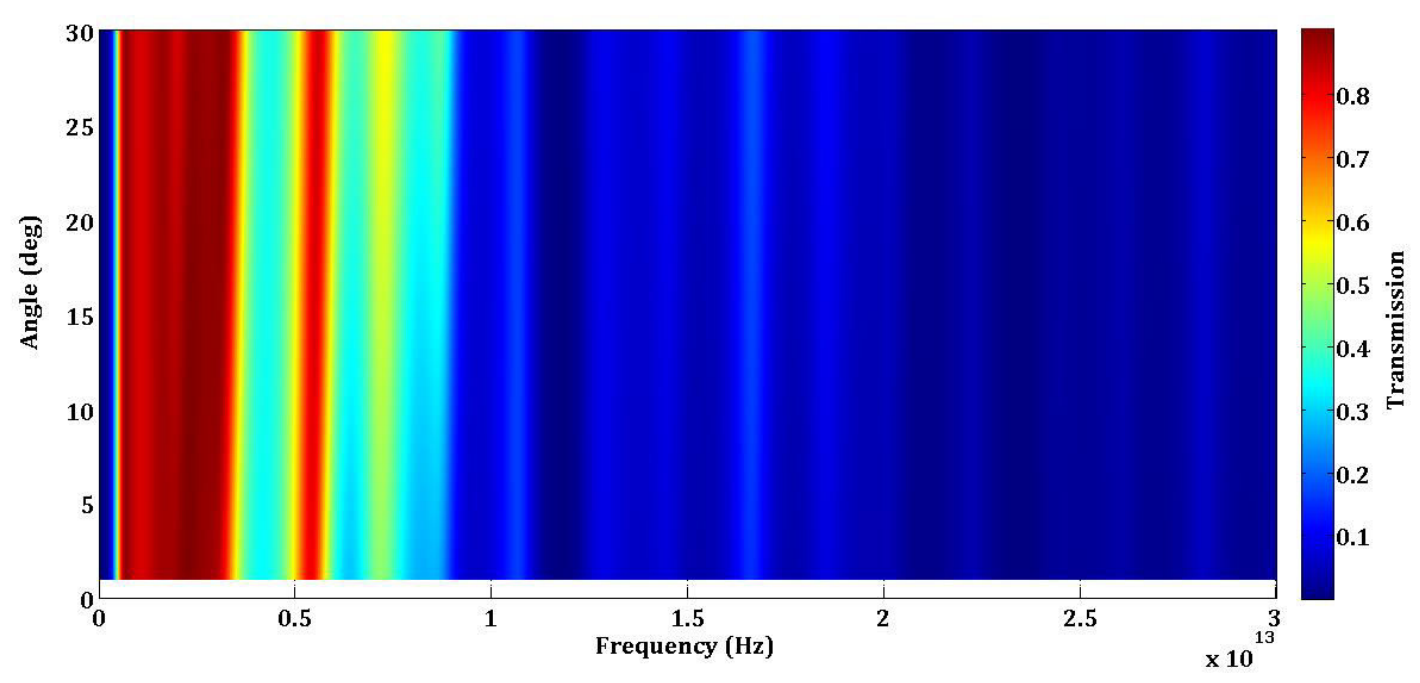

Fig. 8. Transmission of the proposed structure versus different incident angles versus frequency. (Mode A)

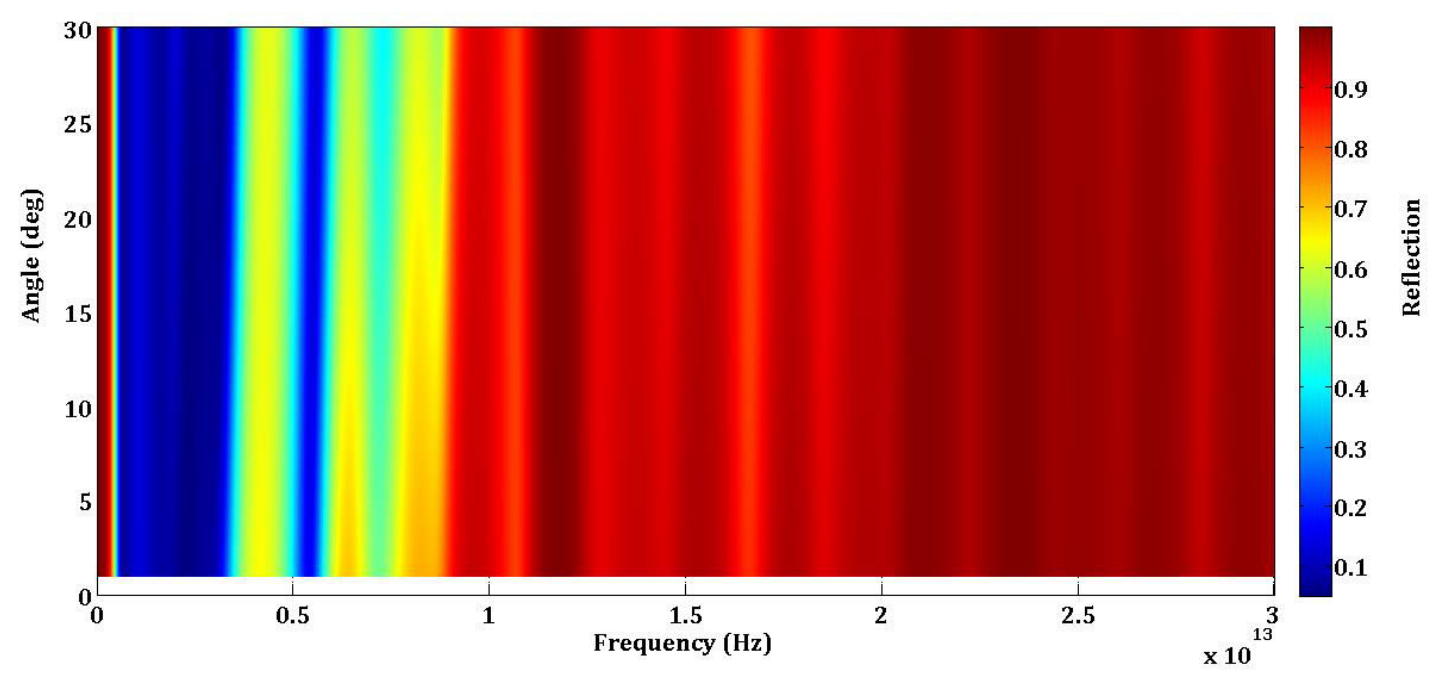

Fig. 9. Reflection of the proposed structure versus different incident angles versus frequency. (Mode A) 


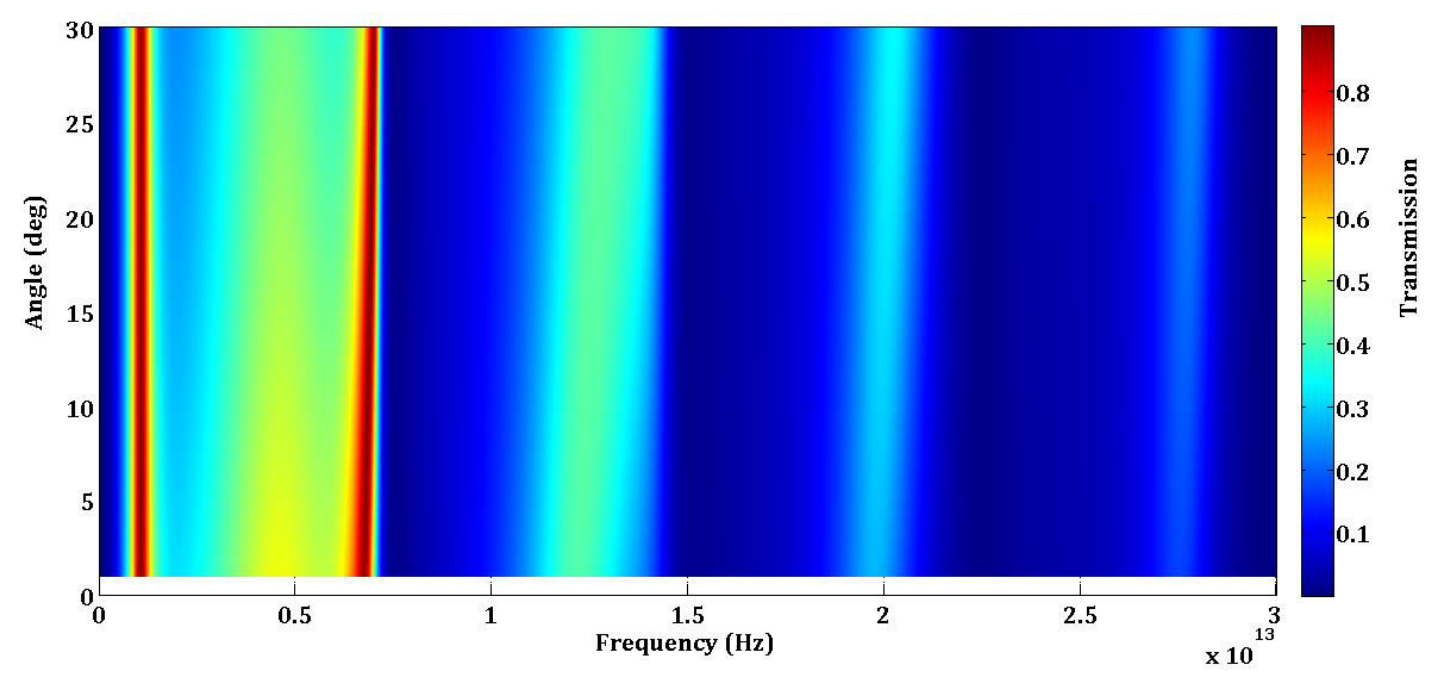

Fig. 10. Transmission of the proposed structure versus different incident angles versus frequency. (Mode B)

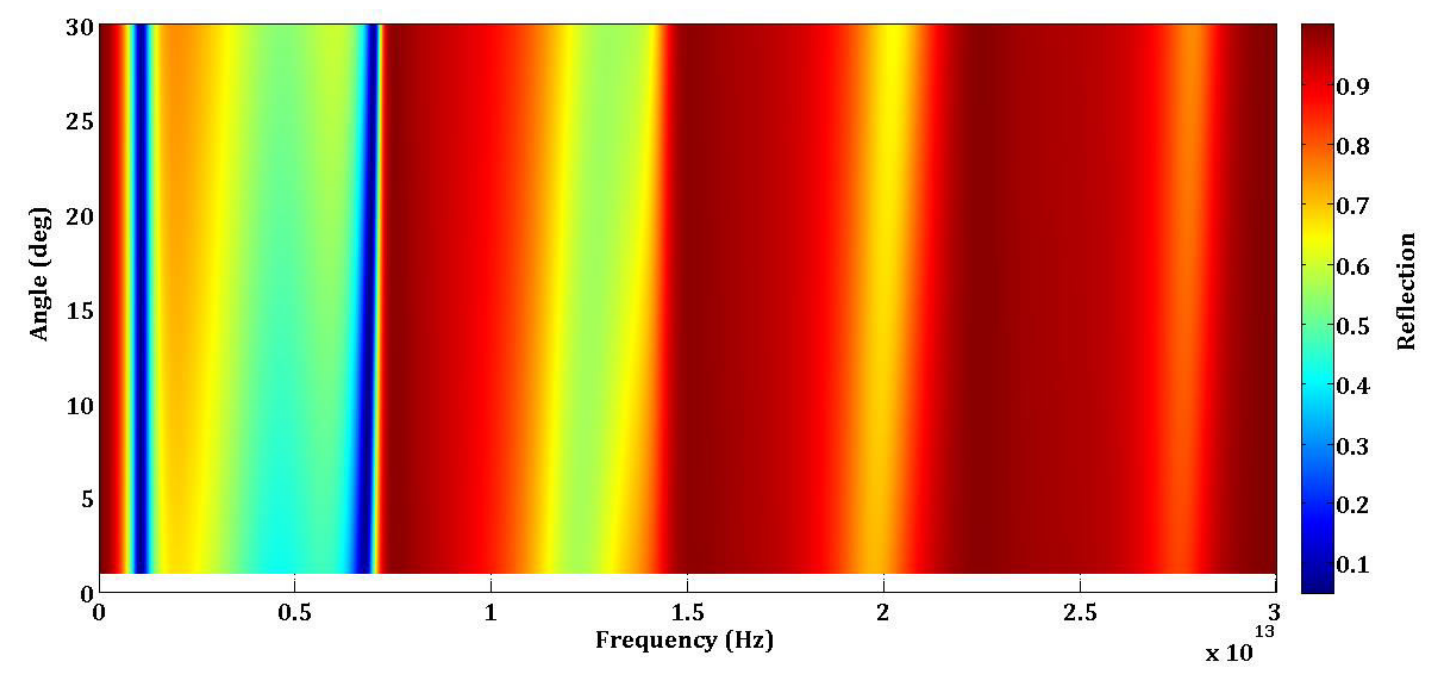

Fig. 11. Reflection of the proposed structure versus different incident angles versus frequency. (Mode A)

Finally, the comparison table is reported in Table 5. 
Table 5. Comparison table.

\begin{tabular}{|c|c|c|c|c|c|c|}
\hline Ref & $\begin{array}{c}\text { Central Frequency } \\
(\mathrm{THz})\end{array}$ & $\begin{array}{c}\text { Absorption } \\
(\%)\end{array}$ & $\begin{array}{c}\text { Transmission } \\
(\%)\end{array}$ & $\begin{array}{c}\text { Reflection } \\
(\%)\end{array}$ & $\begin{array}{c}\text { Total } \\
\text { Height } \\
(\mu m)\end{array}$ & Structure \\
\hline$[24]$ & $0.94,2.69$ & $>95,>83$ & 0 & $<5,<7$ & 95.2 & $\begin{array}{c}\text { Dielectric1 pattern/non- } \\
\text { structured } \\
\text { graphene/dielectric1/metal }\end{array}$ \\
\hline$[25]$ & $1.8,2.4$ & $>95,>95$ & 0 & $<5,<5$ & $\begin{array}{c}\text { square metallic patches/ ultra- } \\
\text { thin insulator dielectric layer/ } \\
\text { continuous metallic ground }\end{array}$ \\
\hline$[26]$ & $3.6,4.7$ & $>90,>85$ & 0 & $<10,<15$ & 120 & $\begin{array}{c}\text { SiO2/Graphene/Polyimide/Si/ } \\
\text { Al2O3/Substrate }\end{array}$ \\
\hline$[27]$ & 188,225 & $>95,>95$ & 0 & $<5,<5$ & 0.77 & $\begin{array}{c}\text { Polymethyl Methacrylate } \\
\text { (PMMA)/Graphene/SiO2/Au }\end{array}$ \\
\hline$[28]$ & 6,25 & $>97,>95$ & 0 & 25 & $\begin{array}{c}\text { Graphene/Polysilicon/Dielectr } \\
\text { ic Slab/Perfect Conductor }\end{array}$ \\
\hline$[29]$ & 3,8 & $>99,>99$ & 0 & $<3,<5$ & $\begin{array}{c}\text { Multi Bias Graphene Ribbons } \\
\text { (twice)/TOPAS (twice)/Au }\end{array}$ \\
\hline $\begin{array}{c}\text { This } \\
\text { Work }\end{array}$ & $\begin{array}{c}\text { Mode A: } 0.6 \text { to } 3.4 \\
\text { Mode B: } 1.03,6.94\end{array}$ & $\begin{array}{c}\text { Mode A: } \sim 4 \\
\text { Mode B: } \sim 4,4\end{array}$ & $\begin{array}{c}\text { Mode A: }>80 \\
\text { Mode B: }>87,>89\end{array}$ & $\begin{array}{c}\text { Mode A: }:<20 \\
\text { Mode B: }<7,<5\end{array}$ & 13 & $\begin{array}{c}\text { Graphene Ribbons/ Sio2/ } \\
\text { Graphene Disks }\end{array}$ \\
\hline
\end{tabular}

\section{Conclusion:}

Using triple-bias graphene patterns of ribbons and disks, a reconfigurable device is presented which can manipulate $\mathrm{THz}$ radiation in terms of transmission, reflection, and absorption. Changing external gate bias stimulates the device to shift operational region against frequency. The device is modeled by circuit model elements to simplify simulation. The sensitivity of the proposed device versus geometrical parameters is reported which verifies the superior performance of the design. Such a reconfigurable device is in great demand for several functions in optical systems. It can be used as a wave duplexer with the capability of tuning via gate biasing.

Data Availability Statement: The data that support the findings of this study are available from the corresponding author upon reasonable request. 


\section{References:}

[1] A. Y. Pawar, D. D. Sonawane, K. B. Erande, and D. V. Derle, "Terahertz technology and its applications," Drug Invention Today, vol. 5, no. 2, pp. 157-163, 2013.

[2] S. Yamaguchi, Y. Fukushi, O. Kubota, T. Itsuji, T. Ouchi, and S. Yamamoto, "Brain tumor imaging of rat fresh tissue using terahertz spectroscopy," Scientific Reports, vol. 6, p. 30124, 2016.

[3] Simpson, Dakotah J., Roberto Gómez-García, and Dimitra Psychogiou. "Single-/multi-band bandpass filters and duplexers with fully reconfigurable transfer-function characteristics." IEEE Transactions on Microwave Theory and Techniques 67.5 (2019): 1854-1869.

[4] I. F. Akyildiz, J. M. Jornet, and C. Han, "Terahertz band: Next frontier for wireless communications," Physical Communication, vol. 12, pp. 16-32, 2014.

[5] Zhuang, Hui, et al. "Duplexer design for full-duplex based wireless communications." China Communications 13.11 (2016): 1-13.

[6] S. Koenig, D. Lopez-Diaz, J. Antes, F. Boes, R. Henneberger, A. Leuther, A. Tessmann, R. Schmogrow, D. Hillerkuss, R. Palmer et al., "Wireless sub-THz communication system with high data rate," Nature Photonics, vol. 7, no. 12, p. 977, 2013.

[7] R. N. Mitra and D. P. Agrawal, "5G mobile technology: A survey," ICT Express, vol. 1, no. 3, pp. 132-137, 2015.

[8] Chen, Liu, et al. "A Novel Notch System Having Two Stopbands Based on Duplexer for Broadband Applications." IEEE Microwave and Wireless Components Letters 26.8 (2016): 577579.

[9] Geim, Andre K., and Konstantin S. Novoselov. "The rise of graphene." Nanoscience and technology: a collection of reviews from nature journals. 2010.11-19.

[10] Khavasi, Amin, and Behzad Rejaei. "Analytical modeling of graphene ribbons as optical circuit elements." IEEE Journal of Quantum Electronics, Vol. 50, no. 6, PP. 397-403, 2014.

[11] Barzegar-Parizi, Saeedeh, Behzad Rejaei, and Amin Khavasi. "Analytical circuit model for periodic arrays of graphene disks." IEEE Journal of Quantum Electronics, Vol. 51, no. 9, PP. 1-7, 2015.

[12] Psychogiou, Dimitra, and Roberto Gómez-García. "Tunable reflectionless microstrip bandpass filters." 2018 IEEE Radio and Wireless Symposium (RWS). IEEE, 2018.

[13] Gómez-García, Roberto, et al. "Wide-band signal-interference duplexer with contiguous single/dual-band channels and its application to quasi-absorptive bandpass filters." Electronics Letters 54.9 (2018): 578-580.

[14] Aghaee, Toktam, and Ali A. Orouji. "Reconfigurable multi-band, graphene-based THz absorber: Circuit model approach." Results in Physics 16 (2020): 102855.

[15] Soltani-Zanjani, Masoud, et al. "Multi-bias graphene-based THz super absorber." Results in Physics 25 (2021): 104326.

[16] Cheng, David Keun. "Field and wave electromagnetics". Pearson Education India, 1989.

[17] Hlali, Aymen, Zied Houaneb, and Hassen Zairi. "Dual-band reconfigurable graphene-based patch antenna in terahertz band: Design, analysis and modeling using WCIP method." Progress In Electromagnetics Research 87 (2018): 213-226.

[18] Chaharmahali, Iman, Sadegh Biabanifard, and Mahdi Mosleh. "Graphene-based multi-layers THz absorber: Circuit model representation." Optik (2020): 165596.

[19] Soltani, Mohamadreza, et al. "A configurable two-layer four-bias graphene-based $\mathrm{THz}$ absorber." Journal of Computational Electronics (2020): 1-17.

[20] Najafi, Alireza, et al. "Reliable design of THz absorbers based on graphene patterns: exploiting genetic algorithm." Optik 203 (2020): 163924. 
[21] Jozani, Kamran Jafari, et al. "Multi-bias, graphene-based reconfigurable $\mathrm{THz}$ absorber/reflector." Optik 198 (2019): 163248.

[22] Zanjani, Masoud Soltani, et al. "A reconfigurable multi-band, multi-bias THz absorber." Optik 191 (2019): 22-32.

[23] Biabanifard, Mohammad, et al. "Analytical design of tunable multi-band terahertz absorber composed of graphene disks." Optik 182 (2019): 433-442.

[24] Qi, Limei, Chang Liu, and Syed Mohsin Ali Shah. "A broad dual-band switchable graphene-based terahertz metamaterial absorber." Carbon 153 (2019): 179-188.

[25] Wang, Ben-Xin, et al. "Design of a dual-band terahertz metamaterial absorber using two identical square patches for sensing application." Nanoscale Advances 2.2 (2020): 763-769.

[26] Cai, Yijun, et al. "Tunable dual-band terahertz absorber with all-dielectric configuration based on graphene." Optics Express 28.21 (2020): 31524-31534.

[27] Wu, Pinghui, et al. "A narrow dual-band monolayer unpatterned graphene-based perfect absorber with critical coupling in the near infrared." Micromachines 11.1 (2020): 58.

[28] Barzegar-Parizi, Saeedeh, and Amin Khavasi. "Designing dual-band absorbers by graphene/metallic metasurfaces." IEEE Journal of Quantum Electronics 55.2 (2019): 1-8.

[29] Aghaee, Toktam, and Ali A. Orouji. "Manipulating pattern periods via external bias for graphene-based THz Dual-Band absorber." Microwave and Optical Technology Letters (2021).

[30] Biabanifard, Sadegh, et al. "Tunable ultra-wideband terahertz absorber based on graphene disks and ribbons." Optics Communications 427 (2018): 418-425.

[31] Sadegh, Biabanifard. "Ultra-broadband terahertz absorber based on graphene ribbons." Optik 172 (2018): 1026-1033.

[32] Biabanifard, M., et al. "Design and comparison of terahertz graphene antenna: ordinary dipole, fractal dipole, spiral, bow-tie and log-periodic." Eng. Technol 2 (2018): 555585.

[33] Biabanifard, Sadegh. "A graphene-based dual-band $\mathrm{THz}$ absorber design exploiting the impedance-matching concept." Journal of Computational Electronics (2020): 1-11.

[34] Cyrus, Sindokht, and Sadegh Biabanifard. "Graphene-based THz absorber: adjustability via multiple gate biasing." Heliyon 7.7 (2021): e07633.

[35] Aghaee, Toktam, and Ali A. Orouji. "Dual-band terahertz absorber based on graphene periodic arrays of disks and ribbons: circuit model approach." Journal of Computational Electronics 20.1 (2021): 611-625.

[36] Aghaee, Toktam, and Ali A. Orouji. "Highly tunable multi-band THz absorber with circuit model representation using multi-bias scheme." International Journal of Numerical Modelling: Electronic Networks, Devices and Fields 33.6 (2020): e2777.

[37] Aghaee, Toktam, and Ali A. Orouji. "Circuit modeling of ultra-broadband terahertz absorber based on graphene array periodic disks." International Journal of Numerical Modelling: Electronic Networks, Devices and Fields 33.3 (2020): e2586. 